\title{
LE FONCTIONNEMENT DES INSTITUTIONS \\ ET LE DÉROULEMENT DES CARRIÈRES DANS LA COLONIE DE PHILIPPES*
}

Faute de pouvoir toujours disposer de documents aussi éloquents que des lois municipales ou des décrets décurionaux, l'étude des institutions des colonies romaines se borne souvent à l'énumération des magistratures attestées dans telle ou telle colonie. Pourtant, en examinant la façon dont ces magistratures s'agencent mutuellement et comment elles s'insèrent dans le reste des institutions, on parvient à appréhender une partie des mécanismes usuels de la vie civique locale. C'est à une analyse de ce genre que nous nous sommes livré pour le cas de la colonie romaine de Philippes, dans la province de Macédoine.

Notre point de départ a été une tentative de tirer profit des carrières municipales telles qu'elles apparaissent dans les cursus honorum épigraphiques. Pour ce faire, nous avons décomposé ces carrières et nous les avons mises en série en vue d'un traitement statistique. Notre but a été d'en dégager des schémas de carrières qui seraient révélateurs de spécificités locales dans le fonctionnement des institutions de la colonie. Par fonctionnement, nous entendons le cours même des institutions, et en particulier le déroulement des magistratures.

Nous sommes conscient des difficultés méthodologiques inhérentes à un tel exercice. Les problèmes qui se posent sont d'ailleurs ceux auxquels on est inévitablement confronté quand on cherche à interpréter une énumération de magistratures dans une inscription : l'ordre dans lequel apparaissent les charges correspond-il à la réalité ? Le cursus est-il ascendant ou descendant ? Toutes les charges revêtues ont-elles été mentionnées ? L'échantillon épigraphique considéré est-il représentatif de pratiques courantes ? De manière générale, nous avons suivi la méthode adoptée par G. Alföldy pour son étude des élites hispaniques et par J. Gascou pour son étude des magistratures municipales de Narbonnaise ${ }^{1}$.

* Les auteurs tiennent à remercier les participants de la rencontre de Paris pour les suggestions qu'ils leur ont faites à l'issue de leur communication. La première partie, consacrée aux filières des carrières philippiennes, a été rédigée par C. Brélaz. La seconde, portant sur la praefectura fabrum, l'a été par A. Rizakis. C. Brélaz a en outre bénéficié, au cours de ses recherches, des remarques de P. Ducrey et R. Frei-Stolba (Lausanne) ainsi que de M. A. Speidel (Berne); qu'ils en soient ici remerciés.

G. Alföldy, "Drei städtische Eliten im römischen Hispanien ", Gerión, 2, 1984, p. 193-238; J. Gascou, "Magistratures et sacerdoces municipaux dans les cités de Gaule Narbonnaise ", dans M. Christol, O. Masson éd., Actes du $X^{\prime}$ Congrès international d'épigraphie grecque et latine, Nîmes, 4-9 octobre 1992, Paris, 1997, p. 75-140. 
Les résultats que nous souhaiterions exposer ici sont provisoires et ils demanderont à être affinés et confirmés. Car le catalogue d'inscriptions mentionnant des magistrats philippiens qui a servi de base à notre étude (au total, plus d'une centaine de numéros, y compris les inscriptions fragmentaires) compte de nombreux inédits. Ces inscriptions inédites seront publiées par l'équipe gréco-franco-suisse travaillant actuellement à la constitution du corpus des inscriptions grecques et latines de la cité macédonienne et colonie romaine de Philippes ${ }^{2}$. C'est pourquoi, dans le cadre de cet aperçu, nous ne fournirons pas de statistiques et renverrons uniquement, à titre indicatif, aux inscriptions de Philippes déjà publiées, qui ont été commodément réunies dans un volume récent par P. Pilhofer ${ }^{3}$. Nous nous contenterons de présenter succinctement les principales observations qui découlent d'un premier examen des carrières des magistrats philippiens connues par le matériel épigraphique ${ }^{4}$.

\section{Une double filière pour la carrière municipale}

On peut dire qu'à Philippes la carrière municipale s'agence schématiquement selon deux filières distinctes. La première, qui est pour ainsi dire la " voie royale ", passe par l'édilité, puis par l'incorporation dans l'ordre des décurions pour aboutir à la charge suprême de la colonie, à savoir le duumvirat ${ }^{5}$. La seconde, en revanche, est manifestement destinée à la frange inférieure de l'élite municipale et on peut l'assimiler à une "voie de garage " ${ }^{6}$ : cette filière passe par une cooptation au sein de l'ordo decurionum avant toute magistrature pour s'interrompre en général à la questure ${ }^{7}$.

Cette tendance à la constitution et à la fixation de deux carrières-types rend compte de la hiérarchie existant à l'intérieur même de l'élite locale :

2 Il s'agit d'un projet commun de l'École française d'Athènes, de l'Éphorie des antiquités préhistoriques et classiques de Kavala, du Centre de recherches de l'Antiquité grecque et romaine (KERA) de la Fondation nationale grecque de la recherche scientifique à Athènes ainsi que de l'Institut d'archéologie et des sciences de l'Antiquité de l'université de Lausanne. Il est placé sous la direction de Ch. Koukouli-Chrysanthaki et de P. Ducrey. Y collaborent notamment A. Bielman, C. Brélaz, G. Duchoud, R. Frei-Stolba, A. D. Rizakis, A. Zannis

3 P. Pilhofer, Plilippi, II. Katalog der Inschriften von Philippi, Tübingen, 2000 (cf. M. B. Hatzopoulos, Bull. ép., 2001, 304). Les numéros cités ci-dessous se rapportent à cette édition.

${ }^{4}$ Les institutions politiques de la colonie de Philippes n'ont pas fait l'objet d'étude particulière depuis l'ouvrage de P. Collart, Philippes, ville de Macédoine, depuis ses origines jusqu'à la fin de l'époque romaine, Paris, 1937, p. 258-274. Pour les sacerdoces publics, cf. M.-D. Poncin, "Les prêtrises publiques dans la colonie de Philippes ", Cahiers Glotz, 12, 2001, p. 229-252.

${ }^{5}$ Cf. $n^{\text {os }} 395,743$.

${ }^{6}$ Nous empruntons l'expression à M. Tarpin (dans Cl. Lepelley éd., Rome et l'intégration de l'Empire, 44 av. J.-C. - 260 apr. J. - C. II. Approches régionales du Haut-Empire romain, Paris, 1998, p. 19-20), qui l'utilise à propos de l'édilité dans la colonie de Venusia, où - à l'inverse de Philippes - c'est la questure qui mène au duumvirat.

${ }^{7}$ Cf. $n^{\circ} 396$. 
1) d'un côté, les notables issus des familles les plus en vue et promis au sommet des honneurs municipaux (duumvirat), entrant à la curie par l'intermédiaire de l'édilité. Cette catégorie de notables est nécessairement minoritaire, puisque deux postes d'édiles seulement sont à pourvoir à nouveau chaque année (pour la questure, voir point 2).

2) de l'autre, les notables de moindre envergure, qui sont cooptés au sein de l'ordo sans passer par l'édilité. Les deux édiles rejoignant chaque année le conseil au sortir de leur charge ne suffisent en effet pas toujours à compenser la mortalité des décurions. Dans ce cas, tous les cinq ans, au moment de revoir la composition du conseil sous la direction des duumvirs quinquennaux ${ }^{8}, \mathrm{l}^{\prime}$ ordo procède à la cooptation de nouveaux décurions n'ayant pas encore exercé de magistrature, afin de garantir l'effectif du conseil. La plupart de ces décurions nouvellement cooptés resteront toutefois des pedani, c'est-à-dire qu'ils seront membres du conseil sans avoir été magistrats". Seule une partie de ceux-ci pourra accéder aux honneurs, le plus souvent à la questure dans le cas de Philippes ${ }^{10}$. En outre, leur carrière s'arrêtera généralement à cet échelon (voir point 2).

Il va de soi que ces types de carrières ne sont que des schémas récurrents et qu'elles n'excluent pas d'autres combinaisons permettant une promotion aux diverses magistratures philippiennes.

\section{Le statut de la questure}

On sait que le statut de la questure n'est pas fixe : la questure n'existe pas dans toutes les colonies et là où elle est présente, elle peut être soit une magistrature ordinaire (honos), soit prendre la forme d'une charge complémentaire $(\text { (munus })^{11}$. La questure est, par ailleurs, une charge financièrement contraignante pour son titulaire, car en tant que responsable des finances municipales, le questeur peut être amené à contribuer sur ses ressources propres aux dépenses de la communauté. C'est pourquoi la questure est souvent une fonction peu recherchée par les notables, sauf s'ils tiennent à manifester leurs bienfaits envers leurs concitoyens.

${ }^{8}$ Cf. $\mathrm{n}^{\text {os }} 26,241$. Il est préférable de résoudre l'abréviation en q(uaestor) plutôt qu'en q(uinquennalis) aux $\mathrm{n}^{\text {os }} 238,239$ et 533 , au vu du déroulement de la carrière des personnages (la succession décurion-questeur est en effet plus naturelle que celle décurion-quinquennal).

${ }^{9}$ Cf. ${ }^{\circ s} 322,433$ (?), 502 (défunt de 58 ans n'ayant été que décurion). Pour les pedani et la hiérarchie en vigueur à l'intéricur de l'ordo, cf. Fr. Jacques, Le privilège de liberté. Politique impériale et autonomie municipale dans les cités de l'Occident romain (161-244), Rome, 1984, en part. p. 477-482.

${ }^{10}$ La dignité de décurion peut aussi être conférée en hommage à un individu ne comptant pas suivre une carrière municipale, conme un sćnateur (cf. $n^{\circ} 61 ;$ même personnage aux $n^{\text {os }}$ 240, 357).

${ }^{11}$ Cf. Char., (sing. de mun. ciu.) dig., 50, 4, 18,2 : Et quaestura in aliqua ciuitate inter honores non habetur, sed personale munus est. 
Il se trouve que la questure est apparemment un munus à Philippes : à l'inverse de l'édilité, elle ne jouit pas du statut de magistrature régulière et ne représente pas une étape ordinaire du cursus honorum. À ce titre, la fonction de questeur, qui est facultative, peut être revêtue indifféremment à un stade ou à un autre de la carrière municipale. Certains notables s'en acquittent au début de leur carrière, parfois en plus de l'édilité ; leur intention, en assumant ce munus, est visiblement d'accélérer leur carrière, car nombre d'entre eux parviendront effectivement au duumvirat ${ }^{12}$. D'autres, probablement les plus fortunés, deviennent questeurs même après le duumvirat, vraisemblablement par évergésie ${ }^{13}$.

De plus, on note dans la colonie une spécialisation de la fonction de questeur : celle-ci semble être, à Philippes, réservée de préférence aux décurions cooptés au sein de l'ordo avant toute magistrature ${ }^{14}$. Comment rendre compte de ce fait ? Une interprétation consisterait à dire que les décurions cooptés sans avoir revêtu de magistrature au préalable, mais désireux toutefois d'entamer une carrière municipale, étaient tenus de s'acquitter de la questure à titre de redevance envers la colonie pour leur promotion directe au sein de l'ordo. Le conseil imposerait cette charge à ceux des pedani qui voudraient devenir magistrats. Comme ces pedani sont entrés dans le conseil sans passer par l'édilité, le fait de leur confier la questure serait en quelque sorte un moyen de leur faire payer leur accession à l'ordo.

\section{La collation des ormements de décurion}

Dans les colonies romaines, le titre de décurion honoraire est en principe octroyé aux individus ne possédant pas les qualités requises pour être décurion de plein droit, mais que l'ordo tient néanmoins à distinguer : ce sont, au premier chef, les affranchis les plus éminents et les fils de notables municipaux n'ayant pas l'àge légal pour entrer à la curie (fixé en principe à vingt-cinq ans). On attribue parfois aussi le décurionat honorifique à des chevaliers ou à des sénateurs connaissant une carrière impériale et que la colonie, à défaut de pouvoir les retenir en permanence sur place, souhaite tout de même célébrer. Ces raisons n'expliquent pourtant que partiellement la très grande fréquence de la pratique de la collation des ornements de décurion à Philippes. Certes, on compte quelques mineurs (les enfants de familles prestigieuses de la colonie) et des chevaliers parmi les décurions honoraires philippiens ${ }^{15}$. Mais le fait que la concession du décurionat honorifique soit si répandue à Philippes

${ }^{12}$ Cf. nos $214,253,438$ (où la questure est même itérée), 719.

${ }^{13}$ Cf. $n^{\circ} 743$. C'est aussi certainement par munificence qu'un chevalier possédant la dignité de décurion a assumé la questure : cf. $\mathrm{n}^{\circ} 718$.

${ }^{14}$ Cf. $n^{\text {os } 396,720 . ~}$

${ }^{15}$ Cf. $n^{\text {os }} 1$ (le chevalier en question, décédé à 23 ans, n'avait toutefois pas encore l'âge requis pour entrer à la curie, d'où les ornements de décurion, et même de duumvir, qui lui avaient été concédés), 492, 493. 
témoigne d'une autre réalité : c'est le reflet d'un déséquilibre démographique entre le nombre élevé de prétendants à l'entrée dans l'ordo et le nombre restreint de places vacantes.

La distinction de décurion honoraire est effectivement offerte aux notables désireux d'entrer à la curie ou de devenir magistrats, mais pour lesquels le nombre de places disponibles est insuffisant. Dans l'attente d'être intégrés de plein droit au sein de l'ordo (que cela se fasse par cooptation directe ou par l'intermédiaire d'une magistrature comme l'édilité), ces notables doivent se contenter des ornements de décurion ${ }^{16}$. On peut dire, en somme, que la collation des ornements de décurion est à Philippes une réponse institutionnelle à une donnée socio-démographique. Devant la pression des notables philippiens ayant l'ambition d'accéder à l'ordo et d'entamer une carrière civique (le grand nombre des prétendants pouvant lui-même s'expliquer par le nombre élevé de colons qui ont été établis à Philippes au moment de la création de la colonie : voir point 7), l'octroi des ornements de décurion constitue un pis-aller temporaire et fonctionne comme une sorte de présélection de l'élite municipale.

\section{La mention de la qualité de décurion dans les cursus honorum épigraphiques}

Les inscriptions qui font connaitre les cursus honorum des notables philippiens (inscriptions honorifiques ou épitaphes) mentionnent régulièrement la qualité de décurion. Dans ces inscriptions, on veille en outre à préciser si le notable a bénéficié de la collation des ornements de décurion au début de sa carrière. Cet usage est à première vue surprenant, car le décurionat - qui n'est pas une magistrature - est souvent omis dans la liste épigraphique des charges revêtues durant leur carrière par les notables des colonies. L'attention avec laquelle l'élite philippienne mentionne le moment auquel la qualité de décurion a été acquise (avant toute magistrature ou, au contraire, à la suite de l'édilité) suggère que l'octroi de la dignité de décurion sert effectivement à Philippes de pivot dans les diverses filières de carrières possibles. Comme nous l'avons évoqué plus haut, la carrière municipale philippienne est en effet essentiellement orientée selon deux filières, qui se distinguent précisément par le moment auquel se fait l'entrée au conseil ${ }^{17}$.

${ }^{16}$ Cf. $\mathrm{n}^{\mathrm{os}} 252,395,396,720$.

${ }^{17}$ Une situation similaire s'observe dans la colonie de Lyon, où la précision du seul décurionat dans une inscription implique que le notable en question est resté un simple pedanus sans accéder aux magistratures ; cf. Fr. Bérard, "L'organisation municipale de la colonie de Lyon ", dans M. Dondin-Payre, M.-Th. Raepsaet-Charlier éd., Cités, Municipes, Colonies. Les processus de municipalisation en Gaule ef en Germanie sous le Haut Empire romain, Paris, 1999, p. 106-107. 


\section{Le titre de munerarius attaché à la magistrature de duumvir}

Il est quasiment automatique que les duumvirs philippiens portent le titre de munerarius ${ }^{18}$. On sait que les magistrats s'acquittent ordinairement d'une summa honoraria à leur entrée en fonction, à titre de compensation envers la communauté pour leur élection. On constate qu'à Philippes cette contribution privée d'intérêt public prend la forme de jeux de gladiateurs (munera) offerts à la population. Ces spectacles, qui se déroulaient au théâtre, réaménagé à cet effet, nous sont connus par de nombreux témoignages épigraphiques, archéologiques et iconographiques à Philippes même ${ }^{19}$. La régularité avec laquelle le titre de munerarius est mentionné aux côtés de la magistrature de duumvir dans les inscriptions montre qu'il était pour ainsi dire institutionnalisé à Philippes que les duumvirs élus, ou peut-être seulement un duumvir au sein de chaque paire, donnent des munera durant leur magistrature. Il ne semble pas toutefois qu'il s'agisse d'une obligation prévue par la loi, comme le prévoit en revanche le règlement municipal de la colonie d'Urso en Bétique, où les duumvirs et les édiles sont contraints d'organiser des jeux de gladiateurs ou des jeux scéniques durant leur magistrature, en dépensant pour cela un minimum légal de mille à deux mille sesterces à leurs frais ${ }^{20}$.

\section{La charge d'irénarque}

On observe, à Philippes, dans le courant du II siècle, l'apparition d'une charge complémentaire et intermittente dans les institutions de la colonie : l'irénarchie ${ }^{21}$. Il s'agit d'une charge municipale de police, ayant formellement le statut de munus, dont s'acquittent les plus grands notables philippiens. L'irénarchie a été introduite dans les institutions municipales sur le modèle de la magistrature grecque d'irénarque (Ei ṕn $\alpha \rho \chi 0 \varsigma$ ), largement diffusée à la même époque dans les cités pérégrines d'Asie Mineure. Philippes n'est pas la seule colonie qui ait procédé à cette innovation, puisque l'irénarchie se rencontre également dans trois colonies romaines d'Anatolie : Antioche de Pisidie, Comama et Iconium. L'exemple de l'irénarchie montre que les colonies, y compris Philippes, ont la capacité de modifier et d'étoffer au besoin leurs institutions.

${ }^{18}$ Cf. $\mathrm{n}^{\text {os }} 252,253,395,493,720$.

${ }^{19}$ P. Collart, Philippes, cit. supra, p. 381-387. Pour les munera et menationes, cf. nº $87,142-144$, 296.

${ }^{20}$ Lex coloniae Genetiuae, LXX-LXXI (M. H. Crawford, Roman Statutes, I, Londres, 1996, p. $\left.393-454, \mathrm{n}^{\circ} 25\right)$. Pour un munus donné par un duumvir patréen à l'occasion de son élection, cf. A. D. Rizakis, Achaïe II. La cité de Patras : épigraphie et histoire, Athènes, 1998, $\mathrm{n}^{\circ} 53$.

${ }^{21}$ Cf. $n^{\text {os }} 120,252+$ deux inédits. Voir C. Brélaz, "Les irénarques de la colonie romaine de Philippes ", dans Actes du XII Congrès international d'épigraphie grecque et latine, Barcelone, 3-8 septembre 2002, à paraître. 


\section{La composition de l'ćlite civique philippienne}

Durant trois siècles - de la double fondation de la colonie par Antoine, puis par Octave, jusqu'aux derniers témoignages épigraphiques mentionnant des magistratures municipales au III siècle -, l'élite civique et politique philippienne reste constituée dans sa grande majorité des descendants des premiers colons d'origine italienne. L'onomastique ne révèle que très peu de familles d'origine indigène (thrace ou grecque) romanisées ou de familles d'anciens affranchis ayant accédé à l'ordo decurionum et aux magistratures de la colonie ${ }^{22}$. Les familles des premiers colons italiens ont de fait monopolisé le pouvoir à Philippes. Cette réticence des notables d'origine italienne à intégrer dans l'ordo des individus ayant obtenu de fraîche date la citoyenneté romaine s'explique sans doute par le nombre probablement élevé de colons installés à Philippes à l'origine. Les descendants de ces colons ont été suffisamment nombreux pour ne pas devoir ouvrir l'ordo à de nouveaux citoyens afin de maintenir l'effectif du conseil.

La puissance de l'implantation italienne à Philippes se traduit notamment par la vigueur du latin, qui perdure comme langue usuelle et officielle de la colonie jusque tard dans le III $^{\circ}$ siècle, à l'inverse des colonies romaines d'Anatolie centrale, où le latin est supplanté plus rapidement par le grec ${ }^{23}$. On peut éventuellement voir une autre manifestation de l'attachement des notables d'origine italienne à leur colonie dans le fait que les magistrats philippiens indiquent régulièrement dans leur titulature le lieu où ils ont accompli leur carrière (en l'occurrence Philippis), bien que la plupart d'entre eux n'ait pas suivi de carrière dans une autre ville ${ }^{24}$. Cette pratique dénote peutêtre, de la part de l'élite locale italienne, une conscience particulièrement affirmée de son identité coloniale et romaine dans un environnement majoritairement pérégrin.

Le type d'analyse que nous avons sommairement appliqué au catalogue des inscriptions des magistrats de Philippes permet d'éclairer les réalités de la vie civique locale. On constate, en particulier, que le fonctionnement des institutions - du moins la configuration des carrières municipales et l'agencement des magistratures entre elles - est largement déterminé par des données sociales et démographiques (répartition de l'élite en plusieurs strates, pression des notables pour entrer dans l'ordo, accaparement du pouvoir par les familles de colons italiens).

22 Cf. $\mathrm{n}^{\text {os }} 322$ (C. Velleius Plato), 396 (M. Antonius Macer), 433 (T. Flauius), 502 (T. Flauii Alexander et Macedonicus). C. Iulius Maximus Mucianus ( $\left.\mathrm{n}^{\circ} 61,240,357\right)$, d'ascendance royale thrace et de rang sénatorial, a été reçu dans l'ordo.Voir F. Mottas, "La population de Philippes et ses origines à la lumière des inscriptions ", EL, 1994, 2, p. 15-24.

${ }^{23}$ B. Levick, Roman Colonies in Southem Asia Minor, Oxford, 1967, p. 130-162. Sur la latinité dans les colonies romaines de Grèce, cf. A. Rizakis, "Le grec face au latin. Le paysage linguistique dans la péninsule balkanique sous l'empire ", dans H. Solin, O. Salomies, U.-M. Liertz éd., Acta colloquii epigraphici Latini Helsingiae 3.-6. sept. 1991 habiti, Helsinki, 1995, p. 373-391.

${ }^{2+}$ Cf. P. Pilhofer, Philippi, cit stupra, p. 881-882 (index 7). 
Les observations qui se dégagent d'un examen statistique des carrières telles qu'elles apparaissent dans les inscriptions ne doivent évidemment pas passer pour des règles, mais pour des tendances. Nous sommes en effet tributaires du matériel épigraphique et de la manière dont les institutions et les filières de carrières se reflètent dans les inscriptions. Néanmoins, la quantité des inscriptions prises en compte et la répétition de mêmes schémas de carrières dans celles-ci permettent de considérer comme valables - en tout cas dans leurs grandes lignes - les résultats indiqués ci-dessus.

$\mathrm{Si}$, à l'avenir, on pouvait répéter le même exercice pour les autres colonies où le corpus épigraphique le permet (quantitativement parlant), on obtiendrait à n'en pas douter une vision plus nuancée du fonctionnement des institutions dans les colonies romaines. Car si les colonies sont toutes dotées d'institutions similaires, inspirées certainement des mêmes archétypes que sont les lois municipales, la façon dont ces institutions sont mises en œuvre varie cependant selon le contexte social propre à chaque colonie. Bien loin d'être des images statiques de Rome, les colonies en sont des ramifications vivantes et autonomes : leurs institutions revêtent une coloration particulière de cas en cas et elles sont susceptibles d'évoluer ${ }^{25}$.

Appendice : la praefectura fabrum, état de la question et exemples philippiens

La praefectura fabrum fait partie de ces fonctions dont on ignore - malgré environ un siècle de recherches - les modalités de l'évolution et le véritable contenu ${ }^{26}$. Au début du $\mathrm{XX}^{\mathrm{e}}$ siècle, $\mathrm{E}$. Kornemann, après avoir résumé les recherches réalisées jusqu'alors, arriva à la conclusion que cette "fonction " avait, sous l'Empire, un caractère civil ${ }^{27}$; d'autres, au contraire, y voyaient une fonction militaire. H.-G. Pflaum, vers le milieu du siècle, pensait que la praefectura fabrum était une distinction honorifique bien appréciée des jeunes chevaliers $^{28}$. D'autres savants soutenaient que le poste n'était rien de plus qu'une

\footnotetext{
${ }^{25}$ Voir les mots fameux d'Aulu-Gelle $(16,13,9): \ldots$ propter amplitudinem maiestatemque populi Romani, cuius istae coloniae quasi effigies paruae simulacraque esse quaedam uidentur. Aulu-Gelle utilise lui aussi la métaphore de la ramification, mais c'est pour signifier que les colonies sont moins libres que les municipia et pour mettre en évidence un lien de dépendance entre elles et Rome $(16,13,8)$ : Non enim ueniunt [scil. les colonies] extrinsecus in ciuitatem nec suis radicibus nituntur, sed ex ciuitate quasi propagatae sunt...

${ }^{26}$ La synthèse la plus récente sur les praefecti fabrum est celle de $M$. Cerva, "La praefectura fabrum : un'introduzione ", dans M. Cébeillac-Gervasoni éd., Les élites municipales de l'Italie péninsulaire de la mort de César à la mort de Domitien, entre continuité ef rupture, classes sociales dirigeantes et pouvoir central. Actes du colloque de Naples du 6 au 8 février 1997, Rome, 2000, p. 177196 (part. p. 177, n. 1 : toute la bibliographie antérieure sur cette question). Sur l'origine de la "fonction ", cf. K. E. Welch, "The Office of Praefectus Fabrum in the Late Republic ", Chiron, 25,1995 , p. $131-145$.

${ }^{27}$ Kornemann, RE,VI, 1909, s. u. Fabri, col. 1923-1924. 218

${ }^{28}$ H.-G. Pflaum, Les procurateurs équestres sous le Haut-Empire romain, Paris, 1950, p. 196-197,
} 
sinécure n'impliquant aucune charge réelle ${ }^{29}$. L'intérêt soulevé par cette question a été renouvelé par le réexamen minutieux de la documentation entrepris par B. Dobson, lequel note les transformations qu'a connues la praefectura depuis son instauration, vers la fin de la République, jusqu'à sa disparition à l'époque des Sévères ${ }^{30}$. Couronnement d'une carrière militaire depuis son instauration jusqu'au règne de Claude, la praefectura perd par la suite tout rapport avec les devoirs militaires, la nomination des préfets pouvant se faire même avant toute forme de service aux armes. D'après B. Dobson, la distinction est attribuée à de jeunes chevaliers ou à des notables locaux et elle est le point de départ d'une carrière équestre. Bien qu'il ne discute que des carrières associées à un cursus militaire, B. Dobson reconnait cependant l'existence d'une praefectura fabrum associée à un cursus purement civil et considère que cette "fonction ", soit comportait quelques devoirs non militaires, soit avait un caractère purement honorifique.

Dès le départ, la difficulté a résidé dans la définition précise du caractère de ces fonctions civiles. Certains savants ont été tentés d'assimiler ces préfets aux praefecti collegiorum fabrum ou à des " magistrats " responsables des incendies, mais, comme on l'a fait remarquer, il est difficile d'admettre que les Romains aient eu deux termes similaires pour désigner des fonctions de nature et d'importance si différentes, à moins de supposer, avec R. Sablayrolles, que la praefectura fabrum était une charge auxiliaire octroyée par un magistrat supérieur ${ }^{31}$. La nature précise de la charge, militaire ou administrative, dépendrait des compétences de la personne elle-même. Cette question n'est malheureusement pas élucidée par la documentation philippienne, dans laquelle cette distinction apparaît à cinq reprises dans des inscriptions funéraires. Dans la plus ancienne, qui daterait du dernier quart du $\mathrm{I}^{\text {er }}$ siècle ap. J.-C., la praefectura fabrum couronne une carrière mixte, militaire et municipale ${ }^{32}$. C. Valerius Valens Vlpianus remplit les fonctions de questeur et de duouir i(ure) d(icundo) - qui font partie du cursus municipal normal à Philippes - après une carrière militaire courte dans les cohortes prétoriennes, à la suite de laquelle il reçoit cet honneur. Deux faits suggèrent une relation étroite avec Vespasien : son second cognomen qui pourrait éventuellement dériver du second nomen, Vlpius,

29 Par exemple F. Millar, dans son compte rendu du livre de H.-G. Pflaum.

${ }^{30}$ B. Dobson, "The Praefectus Fabrum in the Early Principate ", dans M. G. Jarett, B. I obson éd., Britain and Rome. Essays presented to Eric Birley on his sixtieth birthday, Kendal, 1966, p. 61-84 (repris dans D. J. Breczc, B. Dobson éd., Roman Officers and Frontiers, Stuttgart, 1993, p. $218-$ 241).

${ }^{31}$ R. Sablayrolles, "Les pracfecti fabrm dc Narbonnaise ", RAN, 17, 1984, p. 239-247 ; on trouvera dans cet article une présentation rapide de toutes les opinions antérieures concernant cette question.

${ }^{32}$ Ph. Petsas, AEph, 1950/51, p. 58-59, n 4 avec fig. $2(A E, 1952,226 ;$ Th. Sarikakis, Ancient Macedonia, II, Thessalonique, 1977, p. 457, n 208 ; E. Schallmayer, K. Eibl, J. Ott, Corpus der griechischen und lateinischen Beneficiarier-Inschriften, Stuttgart, 1990, p. 520, n 672) ; P. Pilhofer, Philippi, cit. supra, $n^{\circ} 719$ : C. Valerio Valentil / Vlpiano uet(erano) coh(ortis) XI urb(anae) b(eneficiario) q(uaestori) II [uirol / i(ure) d(icundo) Phil(ippis) praef(ecto) fabrum a co(n)s(ule) / flam(ini) diui Vlespasiani ---1. 
de cet empereur ainsi que l'exercice, à Philippes même, du flaminat de Vespasien divinisé. La tribu de C.Valerius Valens n'est pas citée ; il est possible qu'il soit venu d'ailleurs s'installer à Philippes où il s'intégra parmi les notables locaux. Dans un autre exemple, C. Graecinius Firminus, est honoré de la praefectura fabrum, en même temps qu'il a exercé une fonction civile en Afrique, celle de préfet du frumentum mancipale ${ }^{33}$. On peut supposer qu'il est venu s'installer ensuite à Philippes, où il fut décurion de la colonie et où il assuma la questure, probablement non pas dans le cadre d'une carrière municipale, qui n'eut pas de suite, mais comme liturgie, geste de générosité volontaire envers sa nouvelle patrie. Sa carrière municipale fut donc courte, étant donné qu'il n'a pas atteint la fonction suprême de duumvir quinquennal de la colonie. Il en est de même en ce qui concerne sa carrière équestre, qui est fort modeste, puisque Graecinius n'a exercé aucune des fonctions destinées aux membres les plus élevés de cet ordre. Dans deux autres exemples philippiens, la praefectura fabrum est associée à un cursus purement local et prend place soit au milieu de la carrière, comme dans le cas de P. Turpilius Valens ${ }^{34}$, soit à la fin de celle-ci, comme dans le cas de M. Cassius Valens, lequel devait cette distinction honorifique à un consul, comme le précise le texte ${ }^{35}$. Très intéressant enfin est l'exemple de [Ti.] Burrenus Firmus, mort prématurément à l'âge de vingt ans ${ }^{36}$; le personnage porte la distinction de praefectus fabrum, mais on ne saurait dire si elle lui a été transmise en quelque sorte par son père, chevalier romain lui-même, quand il était adolescent ou plus tard ${ }^{37}$.

Les exemples de Philippes montrent que la praefectura ne fait pas partie du cursus local. C'est une distinction attribuée à des notables originaires de Philippes ou domiciliés dans la colonie par un magistrat supérieur, un consul ou le gouverneur de la province, bien que cela ne soit pas toujours précisé.

${ }^{33}$ Ph. Petsas, $A E p h, 1950 / 51$, p. 56-58, n 3 (AE, 1952, 225 ;Th. Sarikakis, Ancient Macedonia, cit. supra, p. 450, $\left.\mathrm{n}^{\circ} 126\right)$; P. Pilhofer, Philippi, cit. supra, $\mathrm{n}^{\circ} 718$ : C(aio) Graecinio C(aii) f(ilio) Vol(tinia) / Firmino praef(ecto) fabrum / et frumenti mancipalis prouinc(iae) Africae / dec(urioni) quaest(ori) col(oniae) Philipp(ensium) an(norum) LVIII / Graeciniae Veneriae an(norum) XLVII / C(aius) Graecinius Romulus Venustus Firmini / [f(ilius) parent(ibus) de s(uis) f(ecit)]. L'administration du frumentum mancipale de l'Afrique est connue par trois inscriptions dont l'une, bilingue, nous donne la traduction de cette fonction en grec : archônès seitou dèmou tôn Rhômaiôn ; elles ont été commentées par M. Christol, "Le blé africain et Rome ", dans Le ravitaillement en blé de Rome, Naples-Rome, 1994, p. 302, n. 45 ; Cl. Nicolet, "Frumentum mancipale" : en Sicile et ailleurs ", dans A. Giovannini éd., Nourrir la plèbe, Bâle, 1991, p. 123-124 ; G. G. Rickman, The Corn Supply of Ancient Rome, Oxford, 1980, p. 84-85.

${ }^{34}$ Ph. Petsas, AEph, 1950/51, p. 55-56, n' 2 (AE, 1952, 224 ; Th. Sarikakis, Ancient Macedonia, cit. supra, p. 456, $\left.\mathrm{n}^{\circ} 190\right)$; P. Pilhofer, Philippi, cit. supra, $\mathrm{n}^{\circ} 717$ : Loco publice dato d(ecreto) d(ecurionum) / P(ublio) Turpilio P(ublii) f(ilio) Volt(inia) Valenti / aed(ili) praefecto fabr(um) II uir(o) i(ure) d(icundo) Phil(ippis) / Pup(us) Turpilius Valens patri.

${ }^{35}$ Cette inscription est encore inédite.

${ }^{36}$ CIL, III/1, 646 ; cf. CIL III/2, p. 989 ad n. 646 (Th. Sarikakis, Ancient Macedonia, cit. supra, p. $447, \mathrm{n}^{\text {os }} 87$ et 89$) ;$ P. Pilhofer, Philippi, cit. supra, $\mathrm{n}^{\circ} 46$.

${ }^{37}$ On trouvera d'autres exemples analogues dans la contribution de M. Cerva, «La praefectura fabrum », cit. supra, p. 190-191. 
L'existence d'un rapport liant ces personnages à l'ordre équestre nous paraît peu probable ${ }^{38}$. Mais on ne pourra nier que cette distinction marque ici, comme ailleurs, la possibilité de passage du plan local aux sphères supérieures des fonctions de l'Etat. Le notable qui se fait remarquer et qui se voit récompensé par ce titre peut espérer accéder à l'ordre équestre. Mais on ne peut pas dire si cette étape est déjà franchie avec la praefectura - c'est le point de vue de R. Sablayrolles - ou si elle reste à franchir. Cette promotion à l'ordre équestre semble rarement s'accomplir pour les porteurs du titre dans les cités provinciales et, en l'occurrence, pour les praefecti fabrum de Philippes. En revanche, on peut dire que la distinction pousse certains de ces praefecti à s'installer dans la capitale provinciale, c'est-à-dire à Thessalonique, d'où proviennent leurs stèles funéraires ${ }^{39}$. Choisis par le gouverneur parmi les notables des villes provinciales, ils résident auprès de lui, mais il est difficile de dire, dans l'état de la documentation actuelle, quel était leur rapport précis avec le gouverneur de la province de Macédoine ${ }^{40}$.

${ }^{38}$ Sur cette question, cf. S. Demougin, L'ordre équestre sous les Julio-Claudiens, Rome, 1988, p. $682-685$.

${ }^{39}$ Ph. Petsas, $A E p h, 1950 / 51$, p. 56-59, nos 3-4 ; p. 62-63, $\mathrm{n}^{\circ} 6$.

${ }^{40}$ Sur cette question, voir en général B. Rankov, "The Govcrnor's Men : the officium consularis in Provincial Administration ", dans A. Goldsworthy, I. Haynes éd., The Roman Army as a Community, Portsmouth, 1999, p. 15-34 ; H. M. Cotton, W. Eck, " Governors and their personnel on latin inscriptions from Caesarea Maritima ", dans Id. éd., Proceedings of The Israel Academy of Sciences and Humanities, VII, Jérusalem, 2001, p. 215-240. 